\title{
SAR determination and influence of the human head in the radiation of a mobile antenna for two different frequencies
}

\author{
Pedro Pinho $^{12}$, Amélia Lopes ${ }^{1}$, João Leite ${ }^{1}$ and João Casaleiro ${ }^{1}$
}

\begin{abstract}
The big proliferation of mobile communication systems has caused an increased concern about the interaction between the human body and the antennas of mobile handsets. In order to study the problem, a multiband antenna was designed, fabricated and measured to operate over two frequency sub bands 900 and $1800 \mathrm{MHz}$. After that, we simulated the same antenna, but now, in the presence of a human head model to analyze the head's influence. First, the influence of the human head on the radiation efficiency of the antenna has been investigated as a function of the distance between the head and the antenna and with the inclination of the antenna. Furthermore, the relative amount of the electromagnetic power absorbed in the head has been obtained.
\end{abstract}

\section{INTRODUCTION}

The cellular phone market has increased substantially during the last decade. With the expansion of current use and anticipated further increases in the use of cellular telephones and other personal communication services, there have been an interest and considerable research effort devoted to interactions between antennas on handsets and the human body. A lot of research studies have been carried out based on this interaction [1-3], dealing generally with two aspects. The first aspect is the mobile phone antenna influence on the human body in particular on the head. In fact, when a cellular phone works, the antenna is near the user's head where a substantial part of radiated power is absorbed. It's important to characterize and quantify the distribution of the electromagnetic fields and the energy levels absorbed by the human tissues. It's important to evaluate the rates of RF energy deposition, called specific absorption rates (SAR), in order to evaluate potential health effects and compliance with standards. SAR is the time rate at which EM energy is absorbed by (dissipated in) an element of biological body mass, and is expressed in units of watts per kilogram $(\mathrm{W} / \mathrm{kg})$ [4]. The maximum local SAR, which is the most critical quantity in the context of potential health effects of RF energy radiated, depends on a large number of factors, such as: design of the device, its operational frequency and antenna input power and orientation with respect to the head.
The second aspect is the influence of the head on radiation properties of the mobile phone. In fact, it's presence close to the handset causes (generally) degradation of its performance in comparison with the operation of the handset alone. Antenna characteristics that are mostly affected by the presence of the human body are radiation pattern, input impedance and radiation efficiency [5]. Its important evaluates deterioration of the antenna performance and develop better antennas, which improve the radiation.

In this paper we study the last two aspects. First, the influence of the human head on the radiation efficiency of the antenna has been investigated as a function of the distance between the head and the handset and with the inclination of the antenna. The considered distances were $1.6 \mathrm{~mm}, 4.4 \mathrm{~mm}$ and 9.6 $\mathrm{mm}$. The antenna's inclinations were $0^{\circ}, 45^{\circ}$, and $90^{\circ}$. In particular, we analyze the changes in the radiation patterns and in the level of return loss, as function of distance and inclination of the handset. Second, we obtained the SAR level for two frequencies, 900 and $1800 \mathrm{MHz}$.

All numerical simulations are performed using a time domain solver based on the FDTD (Finite Difference Time Domain) [6-7] which prove appropriate when handling highly inhomogeneous media such as the human head. Perfectly matched layers (PML) are imposed as boundary absorbing conditions to enclose the simulation domain.

The sections of the present paper are organized as follow. In section 2, we present a multiband antenna that was designed to operate over two frequency sub bands 900 and $1800 \mathrm{MHz}$. The influence of the human head in the radiation proprieties of the antenna at the two frequencies is considered in section 3 . In section 4 the relative amount of the electromagnetic power absorbed in the head has been obtained, calculating the SAR for the two frequencies. Concluding remarks are included in section 5

\section{Patch antenna for $900 / 1800 \mathrm{MHz}$}

\footnotetext{
${ }^{1}$ Instituto Superior de Engenharia de Lisboa, Rua Conselheiro Emidio Navarro 1, 1959-007 Lisboa, Portugal, e-mail: casaleiro@deetc. isel.ipl.pt, tel.: +351218317209, fax: +351218317114

${ }^{2}$ Instituto de Telecomunicações, Campus Universitário de Santiago, 3810 Aveiro, Portugal e-mail: ptpinho@av.it.pt, tel.: +234377900, fax: +234377901.
} 
Patch antennas capable of dual band operations are very suitable for applications in modern mobile communication systems to combat the multipath fading problem, which usually causes larger degradation in the system performance [8]. Here, numerical simulations and measurements of a multi band antenna are presented for GSM 900/1800MHz. The antenna was designed and simulated with the FDTD, and a prototype of the final version was fabricated and measured. To obtain an antenna with two resonances we used a single $U$ slot patch antenna [9] with two short pins, as shown in Figure 1. In, Figure 2 the simulated and measured return loss of the antenna is presented, and a good agree is obtained.
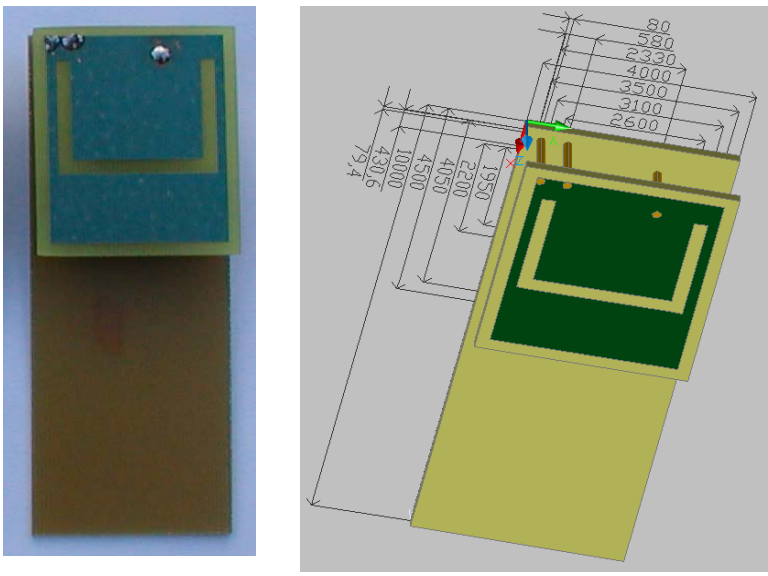

Figure 1 Antenna geometry and dimensions

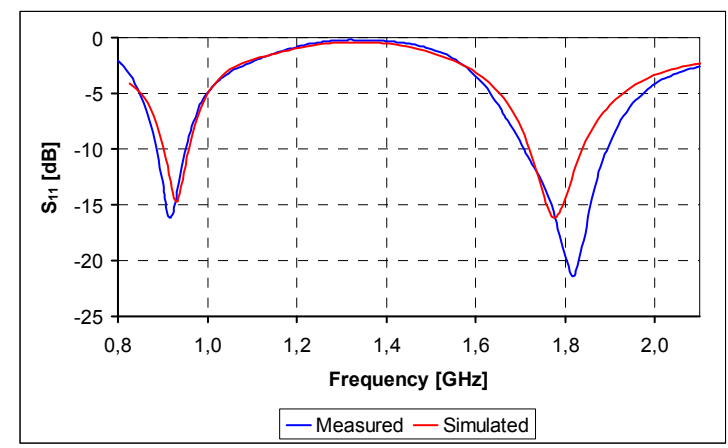

Figure 2: Measured and simulated return loss

\section{Influence of the human head in the radiation proprieties of the antenna}

In this section, we simulated the antenna of Figure 1 , but now, in the presence of a human head model and analyze the head's influence in the radiation pattern and in the return loss. To studying this interaction, it is most important to understand the electromagnetic properties of body tissues. The dielectric properties of body tissues at radio frequencies and microwave frequencies have been examined by several authors. A recent comprehensive study is described in [10]. As a starting point of our research an anatomicallyrealistic model of the human head has been used. It is as most approximate to real heterogeneous human head as recommended in safety standards and uses a grid of $5 \times 5 \times 5 \mathrm{~mm}$.

The antenna's return loss was simulated with a constant distance of $1.6 \mathrm{~mm}$ and inclinations angles of $0^{\circ}, 45^{\circ}$ and $90^{\circ}$. The return loss results are presented on Figure 3.

Analyzing the results it's possible to see that for the $900 \mathrm{MHz}$ there are no significant differences in the level of return loss and on the resonance frequency as well. For the $1800 \mathrm{MHz}$ there is a shift of resonance frequency reducing it about $100 \mathrm{MHz}$. But the level of return loss was improved.

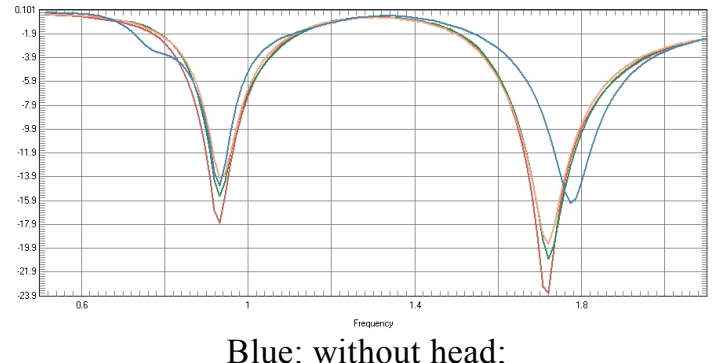

Red: with head at $0^{\circ}$, Green: with head at $45^{\circ}$; Salmon: with head at $90^{\circ}$;

Figure 3: Return loss of the multiband antenna

In Figure 4, we present the simulated results for a constant inclination of $45^{\circ}$ and distances of $1.6 \mathrm{~mm}$, $4.4 \mathrm{~mm}$ and $9.6 \mathrm{~mm}$.

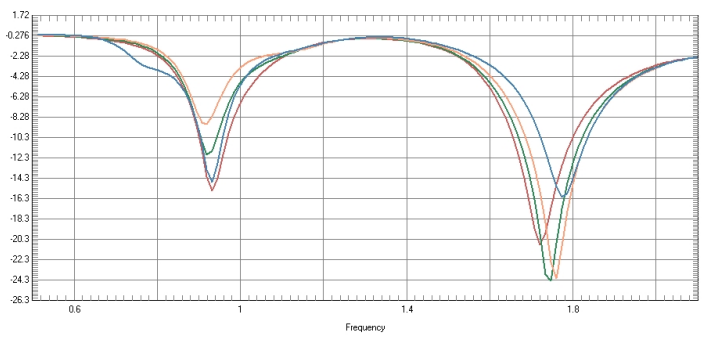

Blue: without head;

Red: with head at $1.6 \mathrm{~mm}$ Green: with head at $4.4 \mathrm{~mm}$ Salmon: with head at $9,6 \mathrm{~mm}$;

Figure 4 Return loss of the multiband antenna

From the last Figure it's possible to see that the antenna is more sensible to distance than to inclination. For $1800 \mathrm{MHz}$ frequency the variation is more accented.

In Figure 5 and Figure 6, we present the radiation patterns of the antenna for $\phi=90^{\circ}$ and for $900 \mathrm{MHz}$. 
In Figure 5 the distance from the antenna to head is constant at $1.6 \mathrm{~mm}$ and the inclination is set to $0^{\circ}$, $45^{\circ}$, and $90^{\circ}$. From Figure it's possible to see the change of radiation pattern, imposed by the presence of the head, for the three different inclinations.

In Figure 6, the antenna inclination is $45^{\circ}$ and the distance is set to: $1.6 \mathrm{~mm}, 4.4 \mathrm{~mm}$ and $9.6 \mathrm{~mm}$. From the Figure is possible to see that the changes in the radiation pattern are less significant. The results obtained for the frequency of $1800 \mathrm{MHz}$ is similar.

Our data suggest that radiation patterns are more sensitive to inclination than to distance. In both situations the head presence changes the radiation pattern, but the unidirectional behavior is maintained.

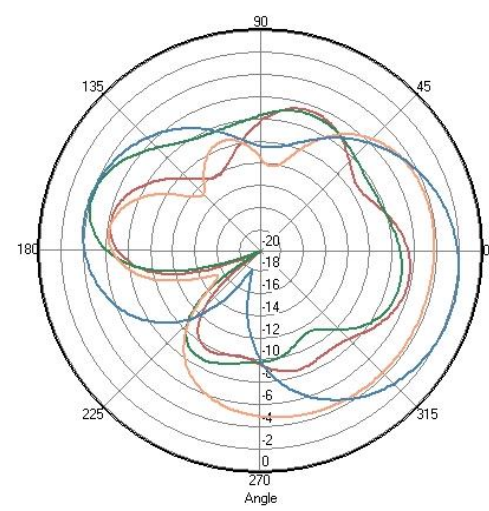

Blue: without head;

Red: with head at $0^{\circ}$ Green: with head at $45^{\circ}$; Salmon: with head at $90^{\circ}$

Figure 5 Radiation pattern of the antenna for $\phi=90^{\circ}$

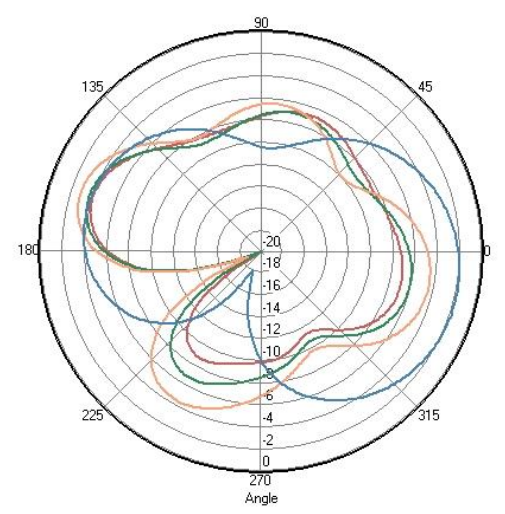

Blue: without head

Red: with head at $1.6 \mathrm{~mm}$ Green: with head at $4.4 \mathrm{~mm}$

Salmon: with head at $9,6 \mathrm{~mm}$

Figure 6 Radiation pattern of the antenna for $\phi=90^{\circ}$

\section{SAR determination}

Various organizations in the world have established safety guidelines for electromagnetic wave absorption. The measurement of this type inside of the human body is extremely complicated and due to this fact, it is imperative the use of numerical methods. The computation of SAR is based on the evaluation of the electromagnetic energy dissipated on the tissue due to an electromagnetic source. Then, this result has to be integrated over a specific volume that is determined on a specific mass. The shape of this volume may be defined in several ways, leading to different possible schemes. In Europe and Australia, the SAR limit for mobile terminal equipment is $2 \mathrm{~mW} / \mathrm{g}$ and measured as an average over a 10 gram cube of tissue. In the USA and Canada, mobile terminal equipment must comply with the $1.6 \mathrm{~mW} / \mathrm{g}$ SAR limit measured as an average in a 1 gram cube of tissue.

In Error! Reference source not found., we present the SAR distribution over the head, for 900 $\mathrm{MHz}$, as a function of handset's inclinations for the same distance of $1.6 \mathrm{~mm}$. The inclinations considered were $0^{\circ}, 45^{\circ}$, and $90^{\circ}$.

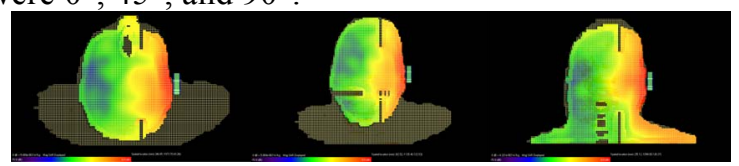

Figure 7: SAR for $900 \mathrm{MHz}$, in the $X o Z$ plane

In Figure 8 we present the same results but now for the frequency of $900 \mathrm{MHz}$.

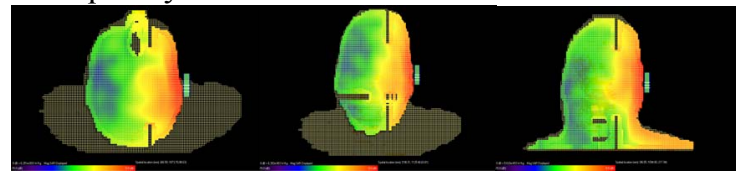

Figure $8 \mathrm{SAR}$ for $1800 \mathrm{MHz}$, in the $X o Z$ plane

Figure 9 presents the SAR distribution over the head, for $900 \mathrm{MHz}$, as a function of handset's distances for an inclination of $45^{\circ}$. The distances considered were $1.6 \mathrm{~mm}, 4.4 \mathrm{~mm}$ and $9.6 \mathrm{~mm}$.

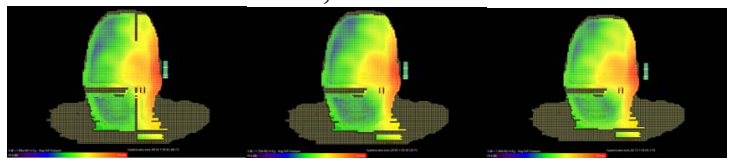

Figure $9 \mathrm{SAR}$ for $900 \mathrm{MHz}$, in the $X o Z$ plane

Figure 10, shows the same results but for the 1800 $\mathrm{MHz}$ frequency.

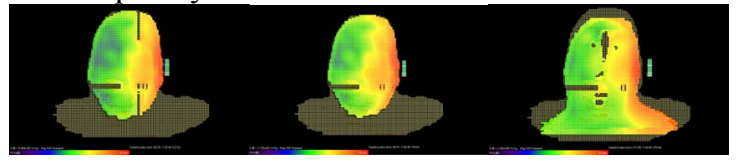

Figure $10 \mathrm{SAR}$ for $1800 \mathrm{MHz}$, in the $X o Z$ plane 
Figure 11 show the SAR as function of the handset inclination for the two frequencies.

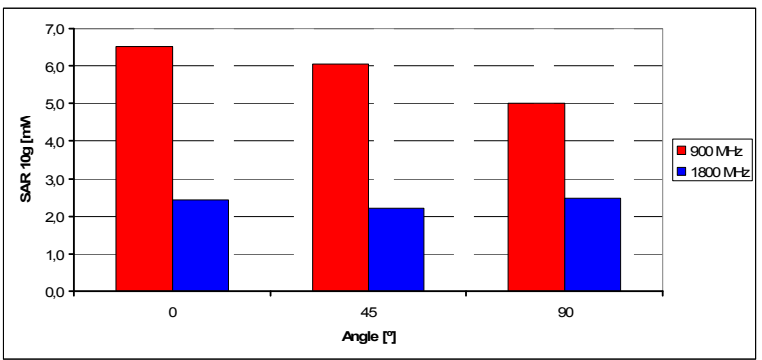

Figure 11 SAR as function of handset inclination.

Figure 12 show the SAR as function of the handset inclination for the two frequencies.

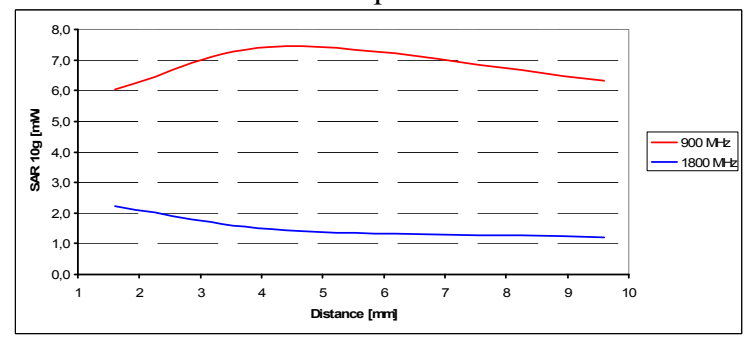

Figure 12 SAR as function of the distance

Investigations have shown that the SAR value depends on excitation frequency. The SAR for 900 $\mathrm{MHz}$ is more than double that obtained for 1800 $\mathrm{MHz}$. There are no significant differences in SAR values between different inclinations of the handset, however when the distance from the head to antenna increase the SAR level tends to decrease but that decrease is not significant.

\section{Conclusions}

In this paper we present the influence of human head in the radiation patterns and in the return loss of a multiband antenna. It's possible to conclude that the radiation patterns have a strong change with the handset inclination, but the omnidirectional behavior remains. Moreover, with the distance there are no significant alterations, but the head presence changes the radiation pattern. In relation to the return loss the human head presence lead a shift in the resonance frequency especially for the $1800 \mathrm{MHz}$. Investigations have shown also that the SAR level depends on excitation frequency, and the handset inclination don't have influence in the SAR level. The SAR level also decreases with the increase of distance.

\section{References}

[1] T. Zervos, A. Alexandridis, V. Petrović, K. Dangakis, B. Kolundžija, D. Olcan, A. Đorđević, C. Soras, "Accurate measurements and modelling of interaction between the human head and the mobile handset", Proc. 7th WSEAS Int. Multiconf. CSCC, 2003.

[2] A. Alexandridis, V. Petrović, K. Dangakis, B. Kolundžija, P. Kostarakis, M. Nikolić, T. Zervos, A. Đorđević, "Accurate modelling and measurements of a mobile handset EM radiation", in Proc. 2nd Intern. Workshop on Biol. Effects of Electromagnetic Fields, pp 251-259,2002.

[3] M.A. Jensen and Y. Rahmat-Samii, "EM interaction of handset antennas and a human in personal communications", Proc. IEEE, vol. 83, pp. 7--17, 1995.

[4] Stevens, N., and L.Martens, "Comparison of Averaging Procedure for SAR Distribution at 900 and $1800 \mathrm{MHz}$," IEEE Transactions on Microwave Theory and Techniques, Vol. 48, no.11, pp. 2180-2184, November 2000.

[5] G. Pedersen, M. Tartiere and M. Knudsen, "Radiation efficiency of handheld phones", in Proc. IEEE Vehicular Technology Conf., Tokyo, Japan, pp. 1381-1385, May 2000.

[6] Bhalla R., Shafai L., Resonance behavior of single U-slot and dual slot antenna, in Antennas and Propagagtion Society International Symposium, 2001, p. 700-703.

[7] K. S. Yee, "Numerical Solution of Initial Boundary Value Problems Involving Maxwell's Equations in isotropic Media”, IEEE Trans. Antennas and Propagation, vol. 14, pp. 302-307, April 1966.

[8] A. Taflove and S. Hagness, Computational Electrodynamics - The Finite - Difference TimeDomain Method, Artech House, 2005.

[9] U. Wahlberg, S. Widell, and C. Beckman, "The performance of polarization diversity antennas at 1800 MHz," in Proc. IEEE Antennas Propagation Soc. Int. Symp. Dig., 1997, pp. 1368-1371.

[10] Gabriel, C., Compilation of the Dielectric Properties of Body Tissues at RF and Microwave Frequencies, Brooks Air Force Technical Report, AL/OE-TR-1996-0037, 1996. 